\title{
GENERALIZED METHOD \\ OF LIE-ALGEBRAIC DISCRETE APPROXIMATIONS FOR SOLVING CAUCHY PROBLEMS WITH EVOLUTION EQUATION
}

\author{
Arkadii Kindybaliuk \\ Ivan Franko National University of Lviv, Lviv, Ukraine \\ a.kindybaluk@mail.ru
}

\begin{abstract}
We consider solving the Cauchy problem with an abstract linear evolution equation by means of the Generalized Method of Lie-algebraic discrete approximations. Discretization of the equation is performed by all variables in equation and leads to a factorial rate of convergence if Lagrange interpolation is used for building quasi representation of differential operator. The rank of a finite dimensional operator and approximation properties have been determined. Error estimations and the factorial rate of convergence have been proved.
\end{abstract}

Keywords: generalized method of Lie-algebraic discrete approximations, dynamical systems, evolution equation, finite dimensional quasi representation, approximations, Lagrange's polynomial, factorial convergence

\section{Introduction}

A wide variety of important environmental processes, problems of techniques, physics are described by ordinary differential equations (ODE) and partial differential equations (PDE). Regardless of powerful mathematical tools, very few of them can be solved exactly. Therefore there is a need for application of either the numerical methods or the analytical-numeric methods. One of these approaches is the method of Lie-algebraic discrete approximations [1]. This method was used by Calogero in 1983 for solving the eigenvalue problem concerning differential equations for spectral problem [2]. Lie algebras and their finite dimensional quasi representations appeared to be very useful in the method devised. Extension of this method for solving PDE was proposed in 1988 and was named as "Lie-algebraic discrete approximation" in [1].

Some history of development of Lie-algebraic method can be found in [3, 4]. The main problem analyzed in these articles is the Cauchy problem for linear evolution equation [3]: 


$$
\left\{\begin{array}{l}
\text { find function } u=u\left(x_{1}, x_{2}, \ldots, x_{q}, t\right) \text { such, that } \\
u_{t}=K(t, x ; \partial) u+f(x, t), x \in \Omega \subset R^{q}, t>0, \\
\left.u\right|_{t=0}=\varphi(x) \in B
\end{array}\right.
$$

where $B$ denotes some functional Banach space.

Similarly, as in Calogero's method, the Heisenberg-Weyl algebra $G=\underset{j=1}{q}\left\{x_{j}, \partial / \partial x_{j}, 1\right\}$ has been used as a basic algebraic tool for constructing the corresponding discrete approximations $X_{j}^{(n)}, Z_{j}^{(n)}, I^{(n)} \in \bigotimes_{j=1}^{q} R^{n_{j}}$.

Using q-dimensional Lagrange interpolation scheme problem (1) is reduced to the Cauchy problem in the following form:

$$
\left\{\begin{array}{l}
\text { find function } u_{(n)}=u_{(n)}(t) \text { such, that } \\
\frac{d u_{(n)}}{d t}=K_{(n)} u_{(n)}+f_{(n)}, t>0 \\
\left.u_{(n)}\right|_{t=0}=\varphi_{(n)} \in B_{(n)}
\end{array}\right.
$$

where $K_{(n)}$ denotes finite dimensional quasi representation of differential operator $K$, and $B_{(n)}$ denotes finite dimensional space of approximations. System (2) is solved by means of Euler's or Runge-Kutta's method [3].

Since reduced problem (2) is solved making use of some numerical algorithm the rate of time convergence is constrained by the convergence rate of the method based on, Lie-algebraic discrete approximations for spatial variables rate of convergence is factorial [1-3].

By means of the Generalized Method of Lie-algebraic discrete approximations proposed in this paper, convergence rate for the time variable becomes factorial.

Moreover, we construct a numerical scheme and prove the convergence of the proposed generalization. We first illustrate the method having applied it to one dimensional case and later we construct its generalization for the multidimensional case.

\section{Cauchy problem and operator equation problem formulation}

We consider a bounded domain $\Omega:=(a, b) \subset R$, time limit $T<+\infty$ and cylinder $Q_{T}=\Omega \times(0, T]$. We assume that linear differential operator $K$ is a formal polynomial of elements from the Lie algebra $\{x, \partial / \partial x, 1\}$ and can be represented as 


$$
K=a_{k} \frac{\partial^{k}}{\partial x^{k}}+a_{k+1} \frac{\partial^{k+1}}{\partial x^{k+1}}+\ldots+a_{k+p} \frac{\partial^{k+p}}{\partial x^{k+p}},
$$

where $a_{k+i} \in R$ for all $i=\overline{0, p}$ and $a_{k} \neq 0$ and $k \geq 1$.

We take the Banach spaces $V=C_{x, t}^{k+p, 1}\left(Q_{T}\right) \cap C\left(\bar{Q}_{T}\right), C=C\left(Q_{T}\right)$ and formulate the Cauchy problem

$$
\left\{\begin{array}{l}
\text { find function } u=u(x, t) \in V \text { such, that } \\
u_{t}=K u+f, \forall(x, t) \in Q_{T}, \\
\left.u\right|_{t=0}=\varphi \in V
\end{array}\right.
$$

where $\varphi=\varphi(x) \in V$ denotes initial conditions and $f=f(x, t) \in C$ represents internal sources.

According to [5], we introduce substitution $u(x, t)=v(x, t)+\varphi(x)$ into (3), which leads to considering the auxiliary Cauchy problem with homogeneous initial condition.

$$
\left\{\begin{array}{l}
\text { find function } v=v(x, t) \in V \text { such, that } \\
v_{t}=K v+K \varphi+f, \forall(x, t) \in Q_{T}, \\
\left.v\right|_{t=0}=0 .
\end{array}\right.
$$

The solution of problem (4) we seek in the subspace of such functions which are homogeneous at the initial moment of time: $B=\left\{v \in V:\left.v\right|_{t=0}=0\right\}$.

Denoting the structure elements in (4) by

$$
A:=\partial / \partial t-K, \tilde{f}=K \varphi+f \in C\left(Q_{T}\right),
$$

we obtain a problem for operator equation:

$$
\left\{\begin{array}{l}
\text { for given operator } A: B \rightarrow C \text { and element } \tilde{f} \in C \\
\text { find element } v \in B \text { such, that } A v=\tilde{f} .
\end{array}\right.
$$

The Cauchy problem has been reduced into the problem for the operator equation. This operator equation we intend to solve by means of the Generalized Method of Lie-algebraic discrete approximations and prove its convergence. 


\section{Numerical scheme and unique of discrete solution}

Let $n_{x}$ denote the count of nodes in domain $\Omega$ and $n_{t}$ denotes count of nodes in interval $[0, T]$. Set of nodes we denote $Q_{T, h}$.

For every variable we construct a set of Lagrange polynomials, which satisfy property $l_{j}\left(x_{i}\right)=\delta_{i j}$ and $l_{j}\left(t_{i}\right)=\delta_{i j}$, where $\delta_{i j}$ denotes Kronecker symbol.

According to the Weierstrass approximation theorem, the set of polynomials with real-valued coefficients is dense set in the space of continuous real-valued functions. Choosing $l(t)=\left\{l_{j}(t)\right\}_{j=2}^{n_{t}}$ we obtain system of polynomials without polynomial associated with initial moment of time. Its easy verifies that $\forall j=\overline{2, n_{t}},\left.l_{j}(t)\right|_{t=0}=0$ and $l(t) \in B$, moreover basis functions $l(t) \otimes l(x) \in B$ are linearly independent, hence system of these functions create basis for approximation spaces $B_{h} \subset B, C_{h} \subset C$. Thus, we seek the solution as a Lagrange interpolation in the following form

$$
v_{h}(x, t)=\sum_{j_{t}=2}^{n_{t}} \sum_{j_{x}=1}^{n_{x}} v_{j} l_{j_{x}}(x) l_{j_{t}}(t)=\bar{v}(l(t) \otimes l(x)),
$$

where $h$ denotes the discretization parameter, $j_{t}$ and $j_{x}$ are indexes of nodes by corresponding variables, $j$ denotes the unique number of the node $j=\left(j_{t}-1\right) n_{x}+j_{x}$ and $\bar{v}$ denotes the set of values $\bar{v}=\left\{v_{j}\right\}_{j=n_{x}+1}^{n_{x} n_{t}}$.

Substitution (7) into equation (6) leads to $A v_{h}=\tilde{f}$ and further using of calculations yields

$$
\left(l^{\prime}(t) \otimes l(x)-l(t) \otimes K(l(x))\right) v_{h}=\tilde{f}_{h} .
$$

Taking $i_{x}=\overline{1, n_{x}}$ and $i_{t}=\overline{2, n_{t}}$ in (8) we obtain a system of linear algebraic equations

$$
\left(Z_{t} \otimes I_{x}-I_{t} \otimes K_{x}\right) \bar{v}=\tilde{f}\left(x_{i_{x}}, t_{i_{t}}\right), i_{x}=\overline{1, n_{x}}, i_{t}=\overline{2, n_{t}} .
$$

Denoting $A_{h}=Z_{t} \otimes I_{x}-I_{t} \otimes K_{x}, \quad$ and $\quad \tilde{f}_{h}=\tilde{f}\left(x_{i_{x}}, t_{i_{t}}\right), i_{x}=\overline{1, n_{x}}, i_{t}=\overline{2, n_{t}} \quad$ we obtain discrete formulation of operator problem

$$
\left\{\begin{array}{l}
\text { for given operator } A_{h}: B_{h} \rightarrow C_{h} \text { and element } \tilde{f}_{h} \in C_{h} \\
\text { find element } v_{h} \in B_{h} \text { such, that } A_{h} v_{h}=\tilde{f}_{h} .
\end{array}\right.
$$


where matrices of corresponding finite dimensional quasi representations have been built upon these rules

$$
Z_{t, i j}=l_{j}^{\prime}\left(t_{i}\right), K_{x, i j}=\left(K l_{j}\right)\left(x_{i}\right), I_{t, i j}=l_{j}\left(t_{i}\right), I_{x, i j}=l_{j}\left(x_{i}\right) .
$$

According to theorem determining the rank of finite dimensional quasi representations [4] we obtain

$$
\operatorname{rank}\left(Z_{t}\right)=n_{t}-1, \operatorname{rank}\left(I_{t}\right)=n_{t}-1, \operatorname{rank}\left(K_{x}\right)=n_{x}-k, \operatorname{rank}\left(I_{x}\right)=n_{x} .
$$

Using property of tensor product we verify that

$$
\operatorname{rank}\left(Z_{t} \otimes I_{x}\right)=n_{x}\left(n_{t}-1\right), \operatorname{rank}\left(I_{t} \otimes K_{x}\right)=\left(n_{t}-1\right)\left(n_{x}-k\right) .
$$

The rank of whole matrix $A_{h}$ remains an open question, and further lemmas give an answer to this question.

Lemma 1. Matrix $\left(Z_{t}^{-1} \otimes K_{x}\right)$ is nilpotent.

Proof. Since $K_{x}$ is finite dimensional quasi representation of operator $\tilde{K}=a_{k} \frac{d^{k}}{d x^{k}}+a_{k+1} \frac{d^{k+1}}{d x^{k+1}}+\ldots+a_{k+p} \frac{d^{k+p}}{d x^{k+p}}$ has the form $K_{x}=a_{k} Z_{x}^{k}+a_{k+1} Z_{x}^{k+1}+\ldots+a_{k+p} Z_{x}^{k+p}$ and matrix $K_{x}$ is nilpotent, hence $\exists m \in N, \forall n \geq m:\left(K_{x}\right)^{n}=0$.

The property of tensor product [6] $\forall n \in N(A \otimes B)^{n}=A^{n} \otimes B^{n}$ yields

$$
\exists m \in N, \forall n \geq m:\left(Z_{t}^{-1} \otimes K_{x}\right)^{n}=\left(Z_{t}^{-1}\right)^{n} \otimes\left(K_{x}\right)^{n}=0,
$$

hence matrix $\left(Z_{t}^{-1} \otimes K_{x}\right)$ is nilpotent.

Lemma 2. Matrix $\left(I_{t} \otimes I_{x}-Z_{t}^{-1} \otimes K_{x}\right)$ has an inverse matrix and its rank is $\left(n_{t}-1\right) n_{x}$ i.e. it has full rank.

Proof. Let us rewrite matrix $\left(I_{t} \otimes I_{x}-Z_{t}^{-1} \otimes K_{x}\right)^{-1}$ as a formal series:

$$
\left(I_{t} \otimes I_{x}-Z_{t}^{-1} \otimes K_{x}\right)^{-1}=\sum_{n=0}^{+\infty}\left(Z_{t}^{-1} \otimes K_{x}\right)^{n}=\sum_{n=0}^{+\infty}\left(Z_{t}^{-1}\right)^{n} \otimes\left(K_{x}\right)^{n}
$$

Since matrices $K_{x},\left(Z_{t}^{-1} \otimes K_{x}\right)$ are nilpotent (Lemma 1) we obtain that the inverse matrix exists because of the existence of finite expansion

$$
\left(I_{t} \otimes I_{x}-Z_{t}^{-1} \otimes K_{x}\right)^{-1}=\sum_{n=0}^{m-1}\left(Z_{t}^{-1} \otimes K_{x}\right)^{n}=\sum_{n=0}^{m-1}\left(Z_{t}^{-1}\right)^{n} \otimes\left(K_{x}\right)^{n} .
$$


However matrix has $n_{x}\left(n_{t}-1\right)$ rows and columns and has inverse matrix, therefore it has full rank: $\operatorname{rank}\left(I_{t} \otimes I_{x}-Z_{t}^{-1} \otimes K_{x}\right)=n_{x}\left(n_{t}-1\right)$.

Using these lemmas we can prove the next theorem.

Theorem 1. The rank of finite dimensional quasi representation $A_{h}$ of operator $A$ has full rank and its rank is $n_{x}\left(n_{t}-1\right)$ and there exists a unique solution of discrete problem (10).

Proof. Let $\quad$ us $\quad$ rewrite $A_{h}=Z_{t} \otimes I_{x}-I_{t} \otimes K_{x}=\left(Z_{t} \otimes I_{x}\right)\left(I_{t} \otimes I_{x}-Z_{t}^{-1} \otimes K_{x}\right)$. However $\operatorname{rank}\left(Z_{t} \otimes I_{x}\right)=n_{x}\left(n_{t}-1\right)$ and due to Lemma $2 \operatorname{rank}\left(I_{t} \otimes I_{x}-Z_{t}^{-1} \otimes K_{x}\right)=$ $=n_{x}\left(n_{t}-1\right)$ using property for two square matrices $A, B: \operatorname{rank}(A B)=$ $=\min \{\operatorname{rank}(A), \operatorname{rank}(B)\}$, yields $\operatorname{rank}\left(Z_{t} \otimes I_{x}-I_{t} \otimes K_{x}\right)=n_{x}\left(n_{t}-1\right)$. Since matrix has full rank then a unique solution of the problem (10) exists there.

\section{Approximation properties of numerical scheme}

According to construction of finite dimensional quasi representation of the operator it can be verified that $\left(A v-A_{h} v\right)_{i}=\left.\left(A v(M)-A v_{I}(M)\right)\right|_{M=M_{i}}$, where $v_{I}$ denotes Lagrange interpolant and $M_{i}=\left(x_{i_{x}}, t_{i_{t}}\right)$ denotes node from $Q_{T, h}$.

Let the dimension of finite dimensional subspaces $B_{h}, C_{h}$ be $\operatorname{dim} B_{h}=\operatorname{dim} C_{h}=N_{h}$. In these spaces we can define the norm in a similar way as it was proposed in [7], namely $\|v\|_{B_{h}}=\|v\|_{C_{h}}=\sqrt{\frac{1}{N_{h}} \sum_{j=1}^{N_{h}} v_{j}^{2}}$.

Assume that $v \in W^{n_{x} n_{t}, \infty}=\left\{v: Q_{T} \rightarrow R: D^{a} v \in L^{\infty}\left(Q_{T}\right), \forall|a| \leq n_{x} n_{t}\right\}$ which means that all possible derivatives till order $n_{x} n_{t}$ are bounded.

The residual of Lagrange interpolation polynomial can be written in the following form [8]:

$$
v(x, t)-v_{I}(x, t)=\frac{\omega_{n_{x}}(x)}{\left(n_{x}\right) !} \frac{\partial^{n_{x}} v(\xi, t)}{\partial x^{n_{x}}}+\frac{\omega_{n_{t}}(t)}{\left(n_{t}\right) !} \frac{\partial^{n_{t}} v(x, \eta)}{\partial t^{n_{t}}}-\frac{\omega_{n_{x}}(x)}{\left(n_{x}\right) !} \frac{\omega_{n_{t}}(y)}{\left(n_{t}\right) !} \frac{\partial^{n_{x}+n_{t}} v\left(\xi_{1}, \eta_{1}\right)}{\partial x^{n_{x}} \partial t^{n_{t}}}
$$

where $\omega_{n_{x}}(x)=\prod_{j=1}^{n_{x}}\left(x-x_{i}\right), \omega_{n_{y}}(y)=\prod_{j=1}^{n_{y}}\left(y-y_{i}\right)$ and $\xi, \xi_{1} \in \Omega, \eta, \eta_{1} \in(0, T)$. 
Let us denote $\alpha$ the highest order of derivative in operator by variable $x$, and $a_{\alpha}$ denotes coefficient standing with highest order of derivative by variable $x$.

Theorem 2. Finite dimensional quasi representation $A_{h}$ of the operator $A$ approximates the operator $A$ on element $v \in B$ and error estimation of approximation has the following form:

$$
\left\|A v-A_{h} v\right\|_{C_{h}} \leq \ln \left(n_{t}\right)\left(\frac{1}{n_{t}-1}\right)^{n_{t}-1}\left\|\frac{\partial^{n_{t}} v}{\partial t^{n_{t}}}\right\|_{\infty}+\left|a_{\alpha}\right|\left(\prod_{k=0}^{\alpha-1}\left(\ln \left(n_{x}-k\right)\right)\right)\left(\frac{1}{n_{x}-1}\right)^{n_{x}-\alpha}\left\|\frac{\partial^{n_{x}} v}{\partial x^{n_{x}}}\right\|_{\infty} .
$$

Proof. Since the norm of space $C_{h}$ is vector norm then according to the construction of finite quasi representation $A_{h}$ of operator $A$ it can verified that

$$
\left(A v-A_{h} v\right)_{i}=\left.\left(A v(M)-A v_{I}(M)\right)\right|_{M=M_{i}},
$$

where $v_{I}$ denotes Lagrange interpolant and $M_{i}=\left(x_{i_{x}}, t_{i_{t}}\right)$ denotes node from $Q_{T, h}$. Using the definition of the norm in space $C_{h}$ after calculations we obtain

$$
\left\|A v-A_{h} v\right\|_{C_{h}} \leq\left\|A v-A v_{I}\right\|_{\infty}=\left\|A\left(v-v_{I}\right)\right\|_{\infty} .
$$

Acting with operator $A$ on residual of Lagrange polynomial we obtain

$$
A\left(v(x, t)-v_{I}(x, t)\right) \approx a_{\alpha} \frac{\omega_{n_{x}}^{(\alpha)}(x)}{\left(n_{x}\right) !} \frac{\partial^{n_{x}} v}{\partial x^{n_{x}}}+\frac{\omega_{n_{t}}^{\prime}(t)}{\left(n_{t}\right) !} \frac{\partial^{n_{t}} v}{\partial t^{n_{t}}} .
$$

Estimation

$$
\left|\omega_{n_{t}}^{\prime}(t)\right| \leq\left(n_{t}\right) ! \ln \left(n_{t}\right)\left(\frac{1}{n_{t}-1}\right)^{n_{t}-1},\left|\omega_{n_{x}-\alpha}(x)\right| \leq\left(n_{x}\right) !\left(\prod_{k=0}^{\alpha-1}\left(\ln \left(n_{x}-k\right)\right)\right)\left(\frac{1}{n_{x}-1}\right)^{n_{x}-\alpha}
$$

and $v \in W^{n_{x} n_{t}, \infty}\left(Q_{t}\right) \cap B$ yields

$$
\left\|A v-A v_{I}\right\|_{\infty} \leq \ln \left(n_{t}\right)\left(\frac{1}{n_{t}-1}\right)^{n_{t}-1}\left\|\frac{\partial^{n_{t}} v}{\partial t^{n_{t}}}\right\|_{\infty}+\left|a_{\alpha}\right|\left(\prod_{k=0}^{\alpha-1}\left(\ln \left(n_{x}-k\right)\right)\right)\left(\frac{1}{n_{x}-1}\right)^{n_{x}-\alpha}\left\|\frac{\partial^{n_{x}} v}{\partial x^{n_{x}}}\right\|_{\infty},
$$

and finally (11) can be obtained. 


\section{Convergence and error estimations}

According to the Kantorovich convergence theorem [9] of abstract approximation scheme $\lim _{h \rightarrow 0}\left\|v-v_{h}\right\|_{B}=0$ holds if

1. there exists a unique solution of equation $A v=\tilde{f}$,

2. for all operators approximating $A_{h}$ operator $A$ exist inverse bounded operators,

3. operator approximates operator $A$ on element $v \in B: \lim _{h \rightarrow 0}\left\|A v-A_{h} v\right\|_{C_{h}}=0$.

The first requirement can be easily verified and the third requirement has been already satisfied in Theorem 2, thus we should prove the second requirement.

Theorem 3. If finite dimensional quasi representation $A_{h}$ of operator $A$ has a full rank and is the same as finite dimensional subspace $B_{h}$ then the bounded inverse operator exists, i.e.:

$$
\forall A_{h}, \exists M>0, \exists A_{h}^{-1}:\left\|A_{h}^{-1}\right\| \leq M<+\infty .
$$

Proof. Although the norm satisfies the axiom of positivity, we obtain $\left\|A_{h} v\right\|_{C_{h}} \geq 0, \forall v \in D\left(A_{h}\right)$ and $\left\|A_{h} v\right\|_{C_{h}}=0 \Leftrightarrow A_{h} v=0_{C_{h}}$.

Let $\left\|A_{h} v\right\|_{C_{h}}=0$, then $A_{h} v=0_{C_{h}}$. For $v \neq 0_{B_{h}}$ it is possible if $\operatorname{det} A_{h}=0$ and $\operatorname{rank} A_{h}<\operatorname{dim} B_{h}$. However, $A_{h}: \operatorname{rank} A_{h}=\operatorname{dim} B_{h}$ then $A_{h} v=0_{C_{h}}$ is possible if $v=0_{B_{h}}$ only. Thus $\forall v \in D\left(A_{h}\right) \backslash\left\{0_{B_{h}}\right\}:\left\|A_{h} v\right\|_{C_{h}}>0$. Since values $\left\|A_{h} v\right\|_{C_{h}}>0$ and $\|v\|_{B_{h}}>0$ are strictly positive for $\forall v \in D\left(A_{h}\right) \backslash\left\{0_{B_{h}}\right\}$ then there exists such constant $\mu>0$ such that $\left\|A_{h} v\right\|_{C_{h}} \geq \mu\|v\|_{B_{h}}$.

According to the theorem of existence of bounded inverse operator [10] setting $M=\frac{1}{\mu}>0$ we obtain (12).

Theorem 4. If $\lim _{h \rightarrow 0}\left\|\tilde{f}-\tilde{f}_{h}\right\|_{C_{h}}=0$ holds and conditions of theorems 1, 2, 3 are satisfied then $\lim _{h \rightarrow 0}\left\|v-v_{h}\right\|_{B_{h}}=0$ and

$$
\left\|v-v_{h}\right\|_{B_{h}} \leq M\left(\ln \left(n_{t}\right)\left(\frac{1}{n_{t}-1}\right)^{n_{t}-1}\left\|\frac{\partial^{n_{t}} v}{\partial t^{n_{t}}}\right\|_{\infty}+\left|a_{\alpha}\right|\left(\prod_{k=0}^{\alpha-1}\left(\ln \left(n_{x}-k\right)\right)\right)\left(\frac{1}{n_{x}-1}\right)^{n_{x}-\alpha}\left\|\frac{\partial^{n_{x}} v}{\partial x^{n_{x}}}\right\|_{\infty}\right)
$$


Proof. Let us consider $\left\|v-v_{h}\right\|_{B_{h}}$ :

$$
\left\|v-v_{h}\right\|_{B_{h}}=\left\|A_{h}^{-1} A_{h}\left(v-v_{h}\right)\right\|_{B_{h}} \leq\left\|A_{h}^{-1}\right\|\left\|A_{h}\left(v-v_{h}\right)\right\|_{C_{h}}
$$

Since the inverse operator is bounded (12) then

$$
\left\|A_{h}^{-1}\right\|\left\|A_{h}\left(v-v_{h}\right)\right\|_{C_{h}} \leq M\left\|A_{h}\left(v-v_{h}\right)\right\|_{C_{h}}
$$

Let us estimate $\left\|A_{h}\left(v-v_{h}\right)\right\|_{C_{h}}:$

$$
\left\|A_{h}\left(v-v_{h}\right)\right\|_{C_{h}}=\left\|A_{h} v-A v+A v-A_{h} v_{h}\right\|_{C_{h}} \leq\left\|A_{h} v-A v\right\|_{C_{h}}+\left\|A v-A_{h} v_{h}\right\|_{C_{h}} .
$$

Since $A v=\tilde{f}$ and $A_{h} v_{h}=\tilde{f}_{h}$, we obtain

$$
\left\|A_{h}\left(v-v_{h}\right)\right\|_{C_{h}} \leq\left\|A_{h} v-A v\right\|_{C_{h}}+\left\|\tilde{f}-\tilde{f}_{h}\right\|_{C_{h}} .
$$

Finally we obtain $\left\|v-v_{h}\right\|_{B_{h}} \leq M\left(\left\|A_{h} v-A v\right\|_{C_{h}}+\left\|\tilde{f}-\tilde{f}_{h}\right\|_{C_{h}}\right)$ and

$$
\lim _{h \rightarrow 0}\left\|v-v_{h}\right\|_{B_{h}} \leq M\left(\lim _{h \rightarrow 0}\left\|A_{h} v-A v\right\|_{C_{h}}+\lim _{h \rightarrow 0}\left\|\tilde{f}-\tilde{f}_{h}\right\|_{C_{h}}\right)=0
$$

Thus $\lim _{h \rightarrow 0}\left\|v-v_{h}\right\|_{B_{h}}=0$.

However $\tilde{f}_{h}$ is Lagrange approximation of $\tilde{f}$, error estimation of $\left\|\tilde{f}-\tilde{f}_{h}\right\|_{B_{h}}$ has the following form: $\left\|\tilde{f}-\tilde{f}_{h}\right\|_{B_{h}} \leq\left(\frac{1}{n_{t}-1}\right)^{n_{t}}\left\|\frac{\partial^{n_{t}} \tilde{f}}{\partial t^{n_{t}}}\right\|_{\infty}+\left(\frac{1}{n_{x}-1}\right)^{n_{x}}\left\|\frac{\partial^{n_{x}} \tilde{f}}{\partial x^{n_{x}}}\right\|_{\infty}$.

Since (11) and $\left(\frac{1}{n_{t}-1}\right)^{n_{t}}$ tends to zero faster than $\left(\frac{1}{n_{t}-1}\right)^{n_{t}-1}$ when $n_{t} \rightarrow \infty$, then neglecting terms with $\left(\frac{1}{n_{t}-1}\right)^{n_{t}}$ and $\left(\frac{1}{n_{x}-1}\right)^{n_{x}}$ yields to error estimation (13).

\section{The Cauchy problem for evolution equation in several dimensions}

The results obtained in previous sections can be generalized in natural way for a multidimensional case. Let us consider q-dimensional bounded domain 
$\Omega:=\left(a_{1}, b_{1}\right) \times\left(a_{2}, b_{2}\right) \times \ldots \times\left(a_{q}, b_{q}\right) \subset R^{q}$, time limit $T<+\infty$ and cylinder $Q_{T}=\Omega \times(0, T]$. Let $\operatorname{diam} \Omega_{i}$ denote the length of the range $\left(a_{i}, b_{i}\right)$. We assume that linear differential operator $K$ is formal polynomial of elements from Lie algebra $\underset{i=1}{q}\left\{x_{j}, \partial / \partial x_{j}, 1\right\}[3,10]$. Let $\alpha_{i}$ denote the highest derivative by variable $x_{i}$ and $a_{i, \alpha}$ denote coefficient standing by the highest derivative by variable $x_{i}$.

Let us consider Banach spaces $V=C_{x_{1}, \ldots, x_{d}, t}^{\alpha_{1}, \ldots, \alpha_{d}, 1}\left(Q_{T}\right) \cap C\left(\bar{Q}_{T}\right), C=C\left(Q_{T}\right)$ and formulation of the Cauchy problem with linear evolution equations [11] is given below

$$
\left\{\begin{array}{l}
\text { find function } u=u\left(x_{1}, \ldots, x_{q}, t\right) \in V \text { such, that } \\
u_{t}=K u+f, \forall\left(x_{1}, \ldots, x_{q}, t\right) \in Q_{T}, \\
\left.u\right|_{t=0}=\varphi \in V
\end{array}\right.
$$

where $\varphi=\varphi\left(x_{1}, \ldots, x_{d}\right) \in V$ denotes initial conditions and $f=f\left(x_{1}, \ldots, x_{q}, t\right) \in C$ represents internal sources.

According to [5] we introduce substitution

$$
u\left(x_{1}, \ldots, x_{q}, t\right)=v\left(x_{1}, \ldots, x_{q}, t\right)+\varphi\left(x_{1}, \ldots, x_{q}\right)
$$

into (14) which leads to considering an auxiliary Cauchy problem with homogeneous initial condition:

$$
\left\{\begin{array}{l}
\text { find function } v=v\left(x_{1}, \ldots, x_{q}, t\right) \in V \text { such, that } \\
v_{t}=K v+K \varphi+f, \forall\left(x_{1}, \ldots, x_{q}, t\right) \in Q_{T}, \\
\left.v\right|_{t=0}=0 .
\end{array}\right.
$$

The solution of problem (15) we seek in the subspace of such functions which are homogeneous at initial moment of time: $B=\left\{v \in V:\left.v\right|_{t=0}=0\right\}$.

Denoting structure elements in (4) by

$$
A:=\partial / \partial t-K, \tilde{f}=K \varphi+f \in C\left(Q_{T}\right),
$$

we obtain the problem for operator equation:

$$
\left\{\begin{array}{l}
\text { for given operator } A: B \rightarrow C \text { and element } \tilde{f} \in C \\
\text { find element } v \in B \text { such, that } A v=\tilde{f} .
\end{array}\right.
$$


The Cauchy problem has been reduced into problem for operator equation. This operator equation we solve by means of Generalized Method of Lie algebraic discrete approximations.

\section{Approximation properties and convergence in multidimensional case}

The numerical scheme for problem (17) is built using dimensional Lagrange interpolation. Let $n_{x_{i}}$ denote the count of nodes by variable $x_{i}$.

Discrete problem is formulated below

$$
\left\{\begin{array}{l}
\text { for given operator } A_{h}: B_{h} \rightarrow C_{h} \text { and element } \tilde{f}_{h} \in C_{h} \\
\text { find element } v_{h} \in B_{h} \text { such, that } A_{h} v_{h}=\tilde{f}_{h} .
\end{array}\right.
$$

Theorem 5. The rank of finite dimensional quasi representation $A_{h}$ of the operator $A$ has a full rank and its rank is $\left(n_{t}-1\right) \prod_{i=1}^{q} n_{x_{i}}$ and there exists a unique solution of the discrete problem (18).

Proof. Using property that finite dimensional quasi representation $K_{h}$ of operator $K$ is nilpotent matrix. Similarly, as in proof of Theorem 1, we obtain that finite dimensional quasi representation has a full rank and hence a unique solution of discrete problem (18) exists there.

Theorem 6. Finite dimensional quasi representation $A_{h}$ approximates operator $A$ on element and error estimation of approximation has the following form

$$
\left\|A v-A_{h} v\right\|_{C_{h}} \leq \ln n_{t}\left(\frac{1}{n_{t}-1}\right)^{n_{i}-1}\left\|\frac{\partial^{n_{h}} v}{\partial t^{n_{t}}}\right\|_{\infty}+\sum_{i=1}^{q}\left(\mid a_{i, \alpha}\left(\left(\prod_{k=0}^{\alpha_{i}-1} \ln \left(n_{x_{i}}-k\right)\right)\left(\frac{1}{n_{x_{i}}-1}\right)^{n_{n_{i}}-\alpha_{i}}\|\| \frac{\partial^{n_{i}} v}{\partial x^{n_{i j}}} \|_{\infty}\right) .\right.
$$

Proof. Using formula of dimensional Lagrange interpolation and acting by operator $A$ on residual yields (19).

Theorem 7. If $\lim _{h \rightarrow 0}\left\|\tilde{f}-\tilde{f}_{h}\right\|_{C_{h}}=0$ holds and conditions of theorems $3,5,6$ are satisfied then $\lim _{h \rightarrow 0}\left\|v-v_{h}\right\|_{B_{h}}=0$ and

$$
\left\|v-v_{h}\right\|_{B_{h}} \leq M\left(\ln n_{t}\left(\frac{1}{n_{t}-1}\right)^{n_{t}-1}\left\|\frac{\partial^{n_{h}} v}{\partial t^{n_{t}}}\right\|_{\infty}+\sum_{i=1}^{q}\left(\mid a_{i, \alpha}\left(\prod_{k=0}^{\alpha_{i}-1} \ln \left(n_{x_{i}}-k\right)\right)\left(\frac{1}{n_{i_{i}}-1}\right)^{n_{n_{i}}-\alpha_{i}}\left\|\frac{\partial^{n_{i_{i}}} v}{\partial x^{n_{i j}}}\right\|_{\infty}\right)\right) .
$$


Proof. According to the Kantorovich convergence theorem of abstract approximation scheme all requirements are satisfied, thus similarly to theorem 4 using inequality $\left\|v-v_{h}\right\|_{B_{h}} \leq M\left\|A v-A_{h} v\right\|_{C_{h}}$ and neglecting terms $\left(\frac{1}{n_{t}-1}\right)^{n_{t}}$ and $\left\{\left(\frac{1}{n_{x_{i}}-1}\right)^{n_{i_{i}}}\right\}_{i=1}^{q}$ we obtain (20).

\section{Conclusions}

Thus, in this paper we present a Generalized Method of Lie algebraic discrete approximations for solving the Cauchy problem for linear dynamical systems. The key finding of this research is the opportunity to provide a factorial rate of convergence by all variables in the equation, including time variable. The Cauchy problem for differential equations has been reduced to a system of linear algebraic equations, which generalize the way of solving ODE and PDE.

Moreover, in the case of linear problem the introduced substitution allows for the rapid solving of the problem when initial data or internal sources have been changed but coefficients of differential operator in the problem remained constant. This is possible by keeping in memory the inverse matrix and multiplying it on the vector which represents initial data or internal sources.

\section{References}

[1] Mytropolskii Yu.A., Prykarpatskii A.K., Samojlenko V.Hr., Algebraic scheme of discrete approximations of linear and nonlinear dynamical systems of mathematical physics, Ukrainian Mathematical Journal 1988, 40, 453-458.

[2] Calogero F., Interpolation, differentiation and solution of eigenvalue problems in more than one dimension, Lett. Nuovo Cimento. 1983, 38, 13, 453-459.

[3] Bihun O., Prytula M., Method of Lie algebraic discrete approximations in the theory of dynamical systems, 2004, 1, 24-31 (in Ukrainian).

[4] Bihun O., Prytula M., The rank of projection-algebraic representations of some differential operators, Matematychni Studii 2011, 35, 1, 9-21.

[5] Kindybaliuk A.A., Prytula M.M., Generalization of scheme of the Lie-algebraic method of discrete approximations for Cauchy problem, XIX Ukrainian Conference of Contemporary Problems of Applied Mathematics and Informatics, L'viv 2013, 73-74 (in Ukrainian).

[6] Horn R.A., Johnson C.R., Matrix Analysis, Cambridge University Press, Cambridge 1990.

[7] Trenogin V.A., Functional Analysis, Fizmatlit, Moscow 2002 (in Russian).

[8] Berezin I.S., Zhydkov N.P., Numeric Methods, Vol. 1, Fizmatgiz, Moscow 1962 (in Russian).

[9] Richtmayer R., Difference Methods of Solving Boundary-value Problems, Mir, Moscow 1972 (in Russian).

[10] Liusternik L.A., Sobolev V.I., Elements of Functional Analysis, Nauka, Moscow 1965 (in Russian).

[11] Samojlenko V.Hr., Algebraic Scheme of Discrete Approximations of Mathematical Physics and its Precision Estimations. Asymptotic Methods in Math. Physics, Mathematics Institute AN USSR, Kyiv 1988, 144-151 (in Russian). 